MATHEMATICS OF COMPUTATION

Volume 68, Number 228, Pages 1325-1346

S 0025-5718(99)01166-7

Article electronically published on May 20, 1999

\title{
CANONICAL CONSTRUCTION OF FINITE ELEMENTS
}

\author{
R. HIPTMAIR
}

\begin{abstract}
The mixed variational formulation of many elliptic boundary value problems involves vector valued function spaces, like, in three dimensions, $\boldsymbol{H}(\operatorname{curl} ; \Omega)$ and $\boldsymbol{H}(\operatorname{Div} ; \Omega)$. Thus finite element subspaces of these function spaces are indispensable for effective finite element discretization schemes.

Given a simplicial triangulation of the computational domain $\Omega$, among others, Raviart, Thomas and Nédélec have found suitable conforming finite elements for $\boldsymbol{H}(\operatorname{Div} ; \Omega)$ and $\boldsymbol{H}(\operatorname{curl} ; \Omega)$. At first glance, it is hard to detect a common guiding principle behind these approaches. We take a fresh look at the construction of the finite spaces, viewing them from the angle of differential forms. This is motivated by the well-known relationships between differential forms and differential operators: div, curl and grad can all be regarded as special incarnations of the exterior derivative of a differential form. Moreover, in the realm of differential forms most concepts are basically dimension-independent.

Thus, we arrive at a fairly canonical procedure to construct conforming finite element subspaces of function spaces related to differential forms. In any dimension we can give a simple characterization of the local polynomial spaces and degrees of freedom underlying the definition of the finite element spaces. With unprecedented ease we can recover the familiar $\boldsymbol{H}(\operatorname{Div} ; \Omega)$ - and $\boldsymbol{H}(\operatorname{curl} ; \Omega)$-conforming finite elements, and establish the unisolvence of degrees of freedom. In addition, the use of differential forms makes it possible to establish crucial algebraic properties of the canonical interpolation operators and representation theorems in a single sweep for all kinds of spaces.
\end{abstract}

\section{INTRODUCTION}

Finite element methods have emerged as an indispensable tool for the computer simulation of large-scale problems of computational physics and engineering. In particular, they are superior to any other approach when complex geometries have to be handled or strong anisotropy of the problem suggests an adaptive policy.

Technically speaking, the finite element method offers a means of discretizing the variational formulation of boundary value problems for partial differential equations. This is usually achieved by specifying a finite dimensional subspace of the continuous function spaces occurring in the variational formulation. After picking a basis, comprising neatly localized basis functions, the discrete problem is converted into a sparse system of equations.

It takes three ingredients to define a finite element space completely (see [8], Chapter 3). First, a triangulation of the computational domain $\Omega \subset \mathbb{R}^{n}, n \in \mathbb{N}$,

Received by the editor January 23, 1997 and, in revised form, November 10, 1997.

1991 Mathematics Subject Classification. Primary 65N30, 41A10, 58A15.

Key words and phrases. Finite elements, differential forms, Raviart-Thomas elements, Nédélec elements, Whitney forms, discrete potentials.

(C)1999 American Mathematical Society 
has to be created. Its individual elements are supposed to have a simple shape. Then for each element a suitable space of polynomials has to be provided, and, after that, a set of global degrees of freedom-linear forms, whose values uniquely characterize a finite element function - must be defined.

A finite element space is called conforming, if it is a proper subspace of the original continuous function space. This can be ensured through enforcing certain coupling conditions across interelement boundaries via a judicious choice of global degrees of freedom.

A special class of finite element schemes are affine families of finite element spaces (see [14, Ch. 2, §2.3]). They rely on the definition of local spaces and degrees of freedom on a reference element. Adequate transformations to general elements, which must not affect the values of degrees of freedom, complete the construction. These spaces are especially convenient for both implementation and analysis.

Much effort has been spent on devising viable finite element schemes for variational problems set in the Sobolev spaces $H^{1}(\Omega)$ and $H^{2}(\Omega)$. Yet, other spaces of vector valued functions like $\boldsymbol{H}(\operatorname{Div} ; \Omega)$ and $\boldsymbol{H}(\operatorname{curl} ; \Omega)$, based on "incomplete" differential operators from vector analysis, are of importance in many physical models, e.g. Maxwell's equations $[6,7]$, and so-called mixed variational formulations (see [11] and [19]).

This paper is devoted to the construction of conforming finite elements for spaces arising from vector analytic differential operators in arbitrary dimension. To keep the presentation simple we confine ourselves to simplicial triangulations. We show how general principles guide the choice of adequate local spaces and degrees of freedom that naturally yield affine families of conforming finite element spaces.

Our approach is the ultimate generalization of a variety of schemes proposed earlier. In [28] Raviart and Thomas introduced $\boldsymbol{H}(\mathrm{Div} ; \Omega)$-conforming finite elements in $2 \mathrm{D}$, which were later dubbed Raviart-Thomas elements. The $3 \mathrm{D}$ case, for both $\boldsymbol{H}(\operatorname{curl} ; \Omega)$ and $\boldsymbol{H}(\operatorname{Div} ; \Omega)$, was dealt with in [26]. The constructions were given for both simplices and parallelepipeds. Extensions to prisms [26, 24] and the isoparametric case followed soon. Several authors proposed slight alterations of the original schemes in order to enhance approximation properties or facilitate implementation $[9,10,27]$.

In a sense, we take our cue from considerations that have been developed in the engineering community $[3,5]$. Motivated by the elegant formulation of Maxwell's equations in the calculus of differential forms $[2,15]$, discretizations were sought that fit this point of view. First order schemes, called Whitney forms, were proposed as conforming finite element methods for spaces of differential forms $[4,25,30]$. In two and three dimensions they agree with Raviart-Thomas and Nédélec elements.

The plan of the paper is as follows:

The next section gives a brief introduction to the concept of differential forms, and discusses their essential features. They are a key instrument for the unified examination of finite element spaces. We summarize the relationships between differential forms and vector fields. The 3D case receives particular scrutiny

In the third section a general recipe for the construction of the local ansatz spaces is presented. We put forward three basic requirements and construct local spaces complying with them. We can confirm that the spaces thus obtained agree with established schemes. 
The forth section explores the issue of suitable degrees of freedom. We arrive at a remarkably simple general formula, and then show that all usual requirements on valid degrees of freedom are met.

The last section deals with the issue of whether finite element functions that belong to the kernel of the differential operator can be represented through discrete potentials. We give a positive answer, proving that a discrete version of Poincaré's lemma holds for the finite element spaces presented in this paper.

\section{Differential FORMS}

Differential forms had been introduced as a trim calculus in differential geometry and analysis. For instance, they pave the way for a unified description of differential operators grad, div, and curl.

Given a $n$-dimensional $\mathbb{R}$-vector space $\mathcal{V}, n \in \mathbb{N}$, we write $\triangle^{l}(\mathcal{V})$ for the space of alternating $l$-multilinear forms $(\mathcal{V})^{l} \mapsto \mathbb{R}$. In addition, we set $\triangle^{0}(\mathcal{V})=\mathbb{R}$. For $l \geq 1$ the space $\triangle^{l}(\mathcal{V})$ has dimension $\left(\begin{array}{l}n \\ l\end{array}\right)$.

Apart from ordinary vector space operations for multilinear forms, a so-called exterior product $\wedge: \triangle^{l}(\mathcal{V}) \times \triangle^{m}(\mathcal{V}) \mapsto \triangle^{m+l}(\mathcal{V})$, a bilinear mapping, is of great importance.

If $\Sigma \subset \mathbb{R}^{n}$ is a $k$-dimensional $C^{1}$-manifold, $1 \leq k \leq n$, and $\boldsymbol{x} \in \Sigma$, we denote by $T_{\Sigma}(\boldsymbol{x})$ the $k$-dimensional space spanned by vectors tangential to $\Sigma$ in $\boldsymbol{x}$.

Definition 1 (Differential forms). A differential form $\omega$ of order $l \in \mathbb{N}_{0}$ defined on a $k$-dimensional, piecewise $C^{1}$ manifold $\Sigma \subset \mathbb{R}^{n}$ is a mapping $\omega: \Sigma \mapsto \triangle^{l}\left(T_{\Sigma}(\cdot)\right)$. If $\Sigma$ is piecewise smooth of class $C^{m+1}$ and this mapping is $m$-times continuously differentiable except maybe on a set of measure zero, it is said to be of class $C^{m}$.

Differential forms of order $l$ form a vector space denoted by $\mathcal{D} \mathcal{F}^{l}(\Sigma)$. For those of class $m$ we write $\mathcal{D} \mathcal{F}^{l, m}(\Sigma)$. Besides, we stick to the convention that $\mathcal{D} \mathcal{F}^{l}(\Sigma)=\{0\}$ if $l>k$ or $l<0$.

If $\Sigma$ coincides with an open domain $\Omega \subset \mathbb{R}^{n}, T_{\Omega}(\boldsymbol{x})=\mathbb{R}^{n}$ for all $\boldsymbol{x} \in \Omega$ is immediate. A basis of $\triangle^{l}\left(\mathbb{R}^{n}\right)$ is given by the set

$$
\left\{d x_{i_{1}} \wedge \ldots \wedge d x_{i_{l}} ; i_{j} \in\{1, \ldots, n\}, 1 \leq j \leq l, i_{1}<i_{2}<\ldots<i_{l}\right\}
$$

of $l$-multilinear forms. The elementary building blocks $\left\{d x_{1}, \ldots, d x_{n}\right\}$ form the canonical dual basis of $\left(\mathbb{R}^{n}\right)^{\prime}$. Thus any $\omega \in \mathcal{D F}^{l}(\Omega)$ has a representation

$$
\omega=\sum_{\left(i_{1}, \ldots, i_{l}\right)} \varphi_{i_{1}, \ldots, i_{l}} d x_{i_{1}} \wedge \ldots \wedge d x_{i_{l}},
$$

where the indices run through all combinations admissible according to (1) and the $\varphi_{i_{1}, \ldots, i_{l}}: \Omega \mapsto \mathbb{R}$ are coefficient functions.

Traces of differential forms on lower dimensional subsets of $\Sigma$ are defined in a straightforward fashion.

Definition 2 (Trace of differential forms). Let $\Gamma \subset \Sigma$ be a piecewise smooth $m$ dimensional manifold contained in the $d$-dimensional $C^{1}$-manifold $\Sigma \subset \mathbb{R}^{n}, 0 \leq$ $m<d \leq n$. For $\omega \in \mathcal{D F}^{l}(\Sigma)$ the trace $\gamma_{\Gamma}(\omega)$, often denoted by $\omega_{\mid \Gamma}$, is an $l$-form on $\Gamma$ given by

$$
\omega_{\mid \Gamma}(\boldsymbol{x}):=\left\{\begin{array}{l}
\left(T_{\Gamma}(\boldsymbol{x})\right)^{l} \mapsto \mathbb{R}, \\
\left(\boldsymbol{t}_{1}, \ldots, \boldsymbol{t}_{l}\right) \mapsto \omega(\boldsymbol{x})\left(\boldsymbol{t}_{1}, \ldots, \boldsymbol{t}_{l}\right), \quad \boldsymbol{x} \in \Gamma .
\end{array}\right.
$$


The three most important concepts in the theory of differential forms are:

- integrals of $l$-forms over piecewise smooth $l$-dimensional manifolds;

- the exterior derivative $d \omega \in \mathcal{D F}^{l+1}(\Omega)$ of a differential form $\omega \in \mathcal{D F}^{l}(\Omega)$;

- the transformation of differential forms $\omega \mapsto \Phi^{*} \omega$ under a change of variable described by a diffeomorphism $\Phi$.

For a comprehensive discussion of the theory of differential forms, see the monograph [13]. We only recall four fundamental theorems establishing close relationships between the above mentioned concepts:

- The so-called Stokes' theorem for differential forms states that

$$
\int_{\partial \Sigma} \omega=\int_{\Sigma} d \omega, \quad \forall \omega \in \mathcal{D F}^{l, 1}(\Sigma)
$$

for an oriented $(l+1)$-dimensional manifold $\Sigma$ with piecewise smooth boundary $\partial \Sigma$.

- Transformations and exterior products commute:

$$
\boldsymbol{\Phi}^{*}(\omega \wedge \sigma)=\boldsymbol{\Phi}^{*} \omega \wedge \boldsymbol{\Phi}^{*} \sigma .
$$

- Transformations and exterior derivatives commute:

$$
d\left(\boldsymbol{\Phi}^{*} \omega\right)=\boldsymbol{\Phi}^{*}(d \omega)
$$

- Integrals are invariant under transformation:

$$
\int_{\Phi(\Omega)} \omega=\int_{\Omega} \Phi^{*} \omega .
$$

For rigorous statements and proofs we refer to [13].

Of course, the differential operator $d$ has no proper inverse. Yet, a sort of partial inverse is supplied by the following potential mapping: Assume that the domain $\Omega \subset \mathbb{R}^{n}$ is star-shaped with respect to $\boldsymbol{a}$. Then, for $l>0$, the linear potential mapping $k_{\boldsymbol{a}}: \mathcal{D F}^{l, 0}(\Omega) \mapsto \mathcal{D} \mathcal{F}^{l-1,0}(\Omega)$ is defined by $\left(\boldsymbol{x} \in \Omega, \boldsymbol{v}_{i} \in \mathbb{R}^{n}\right)$

$$
k_{\boldsymbol{a}}(\omega)(\boldsymbol{x})\left(\boldsymbol{v}_{1}, \ldots, \boldsymbol{v}_{l-1}\right):=\int_{0}^{1} t^{l-1} \omega(\boldsymbol{a}+t(\boldsymbol{x}-\boldsymbol{a}))\left(\boldsymbol{x}-\boldsymbol{a}, \boldsymbol{v}_{1}, \ldots, \boldsymbol{v}_{l-1}\right) d t .
$$

For $\omega \in \mathcal{D F}^{l, 1}(\Omega), k_{\boldsymbol{a}}(\omega)$ is a valid $(l-1)$-form and satisfies (cf. [13], formula 2.13.2)

$$
d\left(k_{\boldsymbol{a}}(\omega)\right)+k_{\boldsymbol{a}}(d \omega)=\omega .
$$

An immediate consequence of this property is the representation theorem:

Theorem 3 (Poincaré's lemma). For a star-shaped domain $\Omega \subset \mathbb{R}^{n}$ every $\omega \in$ $\mathcal{D F}^{l, 1}(\Omega)(l \geq 1)$ with $d \omega=0$ is the differential of an $(l-1)$-form over $\Omega$.

By introducing a basis of the finite dimensional space $\triangle^{l}\left(\mathbb{R}^{n}\right)$ an isomorphism (of vector spaces) between $\triangle^{l}\left(\mathbb{R}^{n}\right)$ and $\mathbb{R}^{D}$ with $D=\left(\begin{array}{c}n \\ l\end{array}\right)$ is established. By the same procedure we can immediately identify $\mathcal{D} \mathcal{F}^{l}(\Omega)$ and vector fields $\Omega \mapsto \mathbb{R}^{D}$.

The basis we are going to choose is that from (1) with the basis functions multiplied by suitable factors \pm 1 . Thus, we get isomorphisms of vector spaces

$$
\Upsilon_{l}^{n}: \mathcal{D F}^{l}(\Omega) \mapsto\left\{\Omega \mapsto \mathbb{R}^{D}\right\}, \quad 0 \leq l \leq n .
$$


The isomorphism (9) permits us to define differential operators $B_{l}^{n}, n \in \mathbb{N}$, $0 \leq l \leq n$, on smooth vector fields by demanding that the diagram

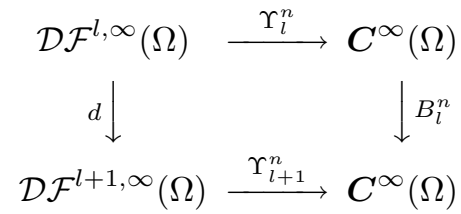

commutes.

Conversely, spaces of differential forms can inherit norms from the related spaces of vector fields. Since the natural habitat of finite element schemes is Hilbert spaces, we introduce an $L^{2}$-norm on $\mathcal{D} \mathcal{F}^{l, \infty}(\Omega)$ by requiring the isomorphisms $\Upsilon_{k}^{n}$ to be isometric:

$$
\|\omega\|_{L^{2}(\Omega)}:=\left\|\Upsilon_{l}^{n}(\omega)\right\|_{L^{2}(\Omega)}
$$

The completion of the space $\mathcal{D} \mathcal{F}^{l, \infty}(\Omega)$ with respect to this norm will be designated by $L_{\mathcal{D} F, l}^{2}(\Omega)$. It goes without saying that the actual norm of a differential form depends on the choice of basis forms. Nevertheless, the definition of the spaces remains the same for all possible bases.

Finite element approximations usually live in spaces of greater smoothness than $L^{2}$. These spaces, designated by $H\left(B_{l}^{n} ; \Omega\right), 0 \leq l \leq n$, arising from norms involving the exterior differential can be obtained as the completion of $\mathcal{D} \mathcal{F}^{l, \infty}(\Omega)$ with respect to the norms

$$
\|\omega\|_{H\left(B_{l}^{n} ; \Omega\right)}^{2}:=\|\omega\|_{L^{2}(\Omega)}^{2}+\|d \omega\|_{L^{2}(\Omega)}^{2} .
$$

Recalling Stokes' theorem for differential forms, we conclude that for differential forms in $H\left(B_{l}^{n} ; \Omega\right), 1 \leq l<n$, their traces on $\partial \Omega$ are well defined $l$-forms in $L_{\mathcal{D} \mathcal{F}, l}^{2}(\partial \Omega)$.

To shed light on these relationships we take a closer look at the case $n=3$. Aiming to retrieve known vector-analytic differential operators as the operators $B_{k}^{3}$, we select the basis $\{1\}$ for $\triangle^{0}$, the basis $\left\{d x_{1}, d x_{2}, d x_{3}\right\}$ for $\triangle^{1}$, the basis $\left\{d x_{2} \wedge d x_{3}, d x_{3} \wedge d x_{1}, d x_{1} \wedge d x_{2}\right\}$ for $\triangle^{2}$, and the basis $\left\{d x_{1} \wedge d x_{2} \wedge d x_{3}\right\}$ for $\triangle^{3}$.

The principal differential operators from vector analysis turn out to be the proper counterparts of the exterior differential. Precisely speaking, we have $B_{0}^{3}=$ grad, $B_{1}^{3}=$ curl, and $B_{2}^{3}=$ div. Similarly, the exterior product of differential forms in $\mathbb{R}^{3}$ is linked to basic operations for vectors by $\Upsilon_{2}^{3}(\omega \wedge \sigma)=\Upsilon_{1}^{3} \omega \times \Upsilon_{1}^{3} \sigma$ for all $\omega, \sigma \in \mathcal{D F}^{1,1}(\Omega)$ and $\Upsilon_{3}^{3}(\omega \wedge \sigma)=\left\langle\Upsilon_{1}^{3} \omega, \Upsilon_{2}^{3} \sigma\right\rangle$ for all $\omega \in \mathcal{D F}^{1,1}(\Omega), \sigma \in \mathcal{D F}^{2,1}(\Omega)$.

Also the transformations of differential forms correspond to familiar transformations. We now assume $\widehat{\Omega}, \Omega \subset \mathbb{R}^{3}$ and consider a $C^{1}$-diffeomorphism $\boldsymbol{\Phi}: \widehat{\Omega} \mapsto \Omega$. Moreover, orientation must be preserved, i.e. $\operatorname{det} D \boldsymbol{\Phi}>0$.

- For 0 -forms $\omega$, which are plain scalar functions, we trivially have

$$
\varphi:=\Upsilon_{0} \omega, \widehat{\varphi}:=\Upsilon_{0} \Phi^{*} \omega \Longrightarrow \hat{\varphi}=(\varphi \circ \boldsymbol{\Phi}) .
$$

- For a 1-form we get, by plugging definitions into each other,

$$
\boldsymbol{\xi}:=\Upsilon_{1} \omega, \widehat{\boldsymbol{\xi}}:=\Upsilon_{1} \boldsymbol{\Phi}^{*} \omega \quad \Longrightarrow \quad \widehat{\boldsymbol{\xi}}=D \boldsymbol{\Phi}^{T}(\boldsymbol{\xi} \circ \boldsymbol{\Phi}) .
$$

So we have recovered the correct transformation formula for the contravariant transformation of $\boldsymbol{H}$ (curl; $\Omega$ )-vector fields presented in [26], formula (5). 
- For 2-forms, tedious manipulations yield

$$
\boldsymbol{u}:=\Upsilon_{2} \omega, \widehat{\boldsymbol{u}}:=\Upsilon_{2} \boldsymbol{\Phi}^{*} \omega \quad \Longrightarrow \quad \widehat{\boldsymbol{u}}=\operatorname{det} D \boldsymbol{\Phi}(D \boldsymbol{\Phi})^{-1}(\boldsymbol{u} \circ \boldsymbol{\Phi}) .
$$

This is exactly Piola's transform (see [28], formula (3.17) and [11], formula (1.45)), a covariant transform of vector fields in $\boldsymbol{H}$ (Div; $\Omega$ ).

- For a 3-form $\omega:=w d x_{1} \wedge d x_{2} \wedge d x_{3}$ the transformation reads

$$
w:=\Upsilon_{3} \omega, \widehat{w}:=\Upsilon_{3} \boldsymbol{\Phi}^{*} \omega \Rightarrow \widehat{w}=\operatorname{det} D \boldsymbol{\Phi}(w \circ \boldsymbol{\Phi}) .
$$

Remark 4. In a finite element environment $\boldsymbol{\Phi}$ might violate $\operatorname{det} D \boldsymbol{\Phi}>0$. To cope with erratic orientations of elements, each transformation formula has to be multiplied by $\operatorname{sgn}(\operatorname{det} D \boldsymbol{\Phi})$.

A straightforward consequence of the commutative property stated in (5) is that, for conventional functions and vector fields alike, transformations and appropriate vector analytic operators are interchangeable.

\section{LOCAL ANSATZ SPACES}

This section introduces suitable local polynomial spaces as the foundation of finite element approximation schemes for the function spaces $H\left(B_{l}^{n}, \Omega\right), 0 \leq l \leq n$. This is the second main ingredient for a valid finite element construction.

Since affine families of finite elements on simplicial meshes are our target, we need only deal with local ansatz spaces on the reference simplex $\widehat{T}$. As usual, $\widehat{T}$ is the simplex spanned by the canonical basis vectors $\boldsymbol{e}_{1}, \ldots, \boldsymbol{e}_{n}$ of $\mathbb{R}^{n} . \mathcal{M}_{i}(\widehat{T})$, $0 \leq i \leq n$, stands for the set of all $i$-dimensional simplices spanned by $i+1$ vertices of $\widehat{T}$. For the local ansatz spaces on the reference element we adopt the notation $\mathcal{X}_{k}^{l}(\widehat{T})$, where $l$ stands for the order of the differential forms and $k \in \mathbb{N}_{0}$ is related to the degree of the polynomials.

Once we have these spaces at our disposal, canonical transformations carry them to an arbitrary element $T$. Formally, the finite element space over $T$ can be described by

$$
\mathcal{X}_{k}^{l}(T):=\boldsymbol{\Phi}^{*} \mathcal{X}_{k}^{l}(\widehat{T}),
$$

where $\boldsymbol{\Phi}: T \mapsto \widehat{T}$ is an affine mapping.

For convenience, we adopt special notations for spaces of "polynomial type" over an $m$-dimensional simplex $S$. A tilde will invariably tag spaces of homogeneous polynomials. For $k, l \in \mathbb{Z}$ we define

$$
\begin{aligned}
\mathcal{P}_{k}(S) & :=\text { Space of } m \text {-variate polynomials of degree } \leq k \text { on } S, \\
\mathcal{D} \mathcal{P}_{k}^{l}(S) & :=\left\{\omega \in \mathcal{D} \mathcal{F}^{l, \infty}(S) ; \varphi_{i_{1}, \ldots, i_{l}} \in \mathcal{P}_{k}(S) \text { in }(2)\right\}, \\
\tilde{\mathcal{P}}_{k}(S) & :=\text { space of } m \text {-variate homogeneous polynomials of degree }=k \text { on } S, \\
\widetilde{\mathcal{D P}}_{k}^{l}(S) & :=\left\{\omega \in \mathcal{D} \mathcal{F}^{l, \infty}(S) ; \varphi_{i_{1}, \ldots, i_{l}} \in \tilde{\mathcal{P}}_{k}(S) \text { in }(2)\right\} .
\end{aligned}
$$

We follow the convention that for $l<0, l>m$ and $k<0$ these spaces are to be trivial. 
First we recall some well-known expressions for the dimensions of polynomial spaces:

$$
\begin{aligned}
\operatorname{dim} \mathcal{P}_{k}(S) & =\left(\begin{array}{c}
m+k \\
k
\end{array}\right), \\
\operatorname{dim} \tilde{\mathcal{P}}_{k}(S) & =\left(\begin{array}{c}
m+k-1 \\
k
\end{array}\right) .
\end{aligned}
$$

Further, we remark that the definition of the spaces $\mathcal{D P}_{k}^{l}(S)$ and $\widetilde{\mathcal{D P}}_{k}^{l}(S)$ is utterly independent of the choice of the basis of $\triangle^{l}\left(T_{S}\right)$. In addition, the space of homogeneous polynomial differential forms belonging to the nullspace of the exterior derivative is of importance:

$$
\widetilde{\mathcal{H N}}_{k}^{l}(S):=\left\{\omega \in \widetilde{\mathcal{D P}}_{k}^{l}(S) ; d \omega=0\right\}
$$

A careful examination of the finite element approaches to $\boldsymbol{H}(\operatorname{Div} ; \Omega)[26,11,28]$ and $\boldsymbol{H}(\operatorname{curl} ; \Omega)[26]$ reveals the following common properties: For $k \in \mathbb{N}_{0}$

$$
\begin{gathered}
d \mathcal{X}_{k}^{l}(\widehat{T}) \subset \mathcal{D} \mathcal{P}_{k}^{l+1}(\widehat{T}), \\
\left\{\omega \in \mathcal{X}_{k}^{l}(\widehat{T}) ; d \omega=0\right\} \subset \mathcal{D} \mathcal{P}_{k}^{l}(\widehat{T}), \\
\mathcal{X}_{k}^{l}(\widehat{T})_{\mid \widehat{f}} \subset \mathcal{X}_{k}^{l}(\widehat{f}) \quad \text { for all "faces" } \widehat{f} \in \mathcal{M}_{n-1}(\widehat{T}) \text { and } l<n .
\end{gathered}
$$

These requirements will guide our construction of $\mathcal{X}_{k}^{l}(\widehat{T})$. Thus, we opt for the following choice of local spaces, which turns out to possess the desired properties:

For a finite element approximation of degree $k \in \mathbb{N}_{0}$, the polynomial space over the reference simplex is given by

$$
\mathcal{X}_{k}^{l}(\widehat{T}):=\mathcal{D P}_{k}^{l}(\widehat{T})+k_{\boldsymbol{a}}\left(\widetilde{\mathcal{H N}}_{k}^{l+1}(\widehat{T})\right)
$$

for an arbitrary $\boldsymbol{a} \in \widehat{T}$. This means that the local finite element spaces are composed of a space of full multivariate polynomials and a potentially incomplete space of polynomials obtained through the potential mapping.

To check the validity of the definition we have to make sure that we end up with the same space $\mathcal{X}_{k}^{l}(\widehat{T})$ for any $\boldsymbol{a}$. For this purpose we need to know that the potential mapping increases the degree of polynomials by no more than one:

Lemma 5. For $\omega \in \widetilde{\mathcal{D P}}_{k}^{l}(\widehat{T}), k>0$, we know that $d \omega \in \mathcal{D P}_{k-1}^{l+1}(\widehat{T})$ and $k_{\boldsymbol{a}}(\omega) \in$ $\mathcal{D} \mathcal{P}_{k+1}^{l-1}(\widehat{T})$. In the special case $\boldsymbol{a}=0$, the even stronger assertion $k_{0}(\omega) \in \widetilde{\mathcal{D P}}_{k+1}^{l-1}(\widehat{T})$ holds true.

Proof. (I) Ordinary differentiation reduces the degree of a polynomial by exactly one. Since the exterior derivative involves first order derivatives of the coefficient functions of a differential form, the first assertion is obvious.

(II) By virtue of the linearity of the potential mapping, we only have to establish the second assertion for $\omega$ of the form

$$
\omega=p(\boldsymbol{x}) d x_{i_{1}} \wedge \ldots \wedge d x_{i_{l}},
$$


where $p \in \tilde{\mathcal{P}}_{k}(\Omega)$. Citing the definition of the inner product of differential forms, we have, with $\boldsymbol{v}_{i}=\left(v_{i, 1}, \ldots, v_{i, n}\right)^{T} \in \mathbb{R}^{n}, i=1, \ldots, l-1$,

$$
\begin{aligned}
\omega(t \boldsymbol{x} & +\boldsymbol{c})\left(\boldsymbol{x}-\boldsymbol{a}, \boldsymbol{v}_{1}, \ldots, \boldsymbol{v}_{l-1}\right) \\
& =p(t \boldsymbol{x}+\boldsymbol{c}) \cdot \operatorname{det}\left(\begin{array}{cccc}
x_{i_{1}}-a_{i_{1}} & v_{1, i_{1}} & \ldots & v_{l-1, i_{1}} \\
\vdots & \vdots & & \vdots \\
x_{i_{l}}-a_{i_{l}} & v_{1, i_{l}} & \ldots & v_{l-1, i_{l}}
\end{array}\right) .
\end{aligned}
$$

By the recursion formula for determinants this expresssion is equal to

$$
\begin{aligned}
& \omega(t \boldsymbol{x}+\boldsymbol{c})\left(\boldsymbol{x}-\boldsymbol{a}, \boldsymbol{v}_{1}, \ldots, \boldsymbol{v}_{l-1}\right) \\
& =p(t \boldsymbol{x}+\boldsymbol{c}) \sum_{k=1}^{l}(-1)^{k}\left(x_{i_{k}}-a_{i_{k}}\right) \cdot \operatorname{det}\left(\begin{array}{ccc}
v_{1, i_{1}} & \ldots & v_{l-1, i_{1}} \\
\vdots & & \vdots \\
\check{v}_{1, i_{k}} & \ldots & \check{v}_{l-1, i_{k}} \\
\vdots & & \vdots \\
v_{1, i_{l}} & \ldots & v_{l-1, i_{l}}
\end{array}\right),
\end{aligned}
$$

where the ${ }^{\sim}$ marks a line of the matrix which has to be omitted. Thus we see that, with the token $\bullet$ standing for $\boldsymbol{v}_{1}, \ldots, \boldsymbol{v}_{l-1}$,

$$
\begin{aligned}
\omega(t \boldsymbol{x} & +\boldsymbol{c})(\boldsymbol{x}-\boldsymbol{a}, \bullet) \\
& =\sum_{k=1}^{l} \underbrace{(-1)^{k} p(t \boldsymbol{x}+\boldsymbol{c})\left(x_{i_{k}}-a_{i_{k}}\right)}_{\in \mathcal{P}_{k+1}(\Omega)} d x_{i_{1}} \wedge \ldots \wedge \check{d x_{i_{k}}} \wedge \ldots \wedge d x_{i_{l}}(\bullet) .
\end{aligned}
$$

Plugging this into the definition (7) of the potential mapping $k_{\boldsymbol{a}}(\omega)$ gives the second contention.

In the case $\boldsymbol{a}=0, \boldsymbol{c}=0$ the above expression reduces to

$$
\omega(t \boldsymbol{x})(\boldsymbol{x}, \bullet)=t^{k} \sum_{k=1}^{l} \underbrace{(-1)^{k} p(\boldsymbol{x}) x_{i_{k}}}_{\in \tilde{\mathcal{P}}_{k+1}(\Omega)} d x_{i_{1}} \wedge \ldots \wedge \check{d x_{i_{k}}} \wedge \ldots \wedge d x_{i_{l}}(\bullet) .
$$

Thus, the coefficient functions of $k_{0}(\omega)$ remain homogeneous polynomials.

Now we are able to show that the definition (16) is independent of $\boldsymbol{a}$. First recall that for $p \in \mathcal{P}_{k}(\widehat{T})$ the difference $p(\cdot)-p(\cdot-\boldsymbol{c})$ belongs to $\mathcal{P}_{k-1}(\widehat{T})$ for any $\boldsymbol{c} \in \mathbb{R}^{n}$. Therefore we can decompose $\omega \in \mathcal{D P}_{k}^{l}(\widehat{T})$ in

$$
\omega(\boldsymbol{a}+t(\boldsymbol{x}-\boldsymbol{a}))=\omega(t \boldsymbol{x})+\pi(t \boldsymbol{x}),
$$

where $\pi \in \mathcal{D} \mathcal{P}_{k-1}^{l}(\widehat{T})$. Using the definition of the potential mapping, we get

$$
\begin{aligned}
& k_{\boldsymbol{a}}(\omega)(\boldsymbol{x})(\bullet) \\
& =-\int_{0}^{1} t^{l-1} \underbrace{\omega(\boldsymbol{a}+t(\boldsymbol{x}-\boldsymbol{a}))(\boldsymbol{a}, \bullet)}_{\in \mathcal{D P}_{k}^{l-1}(\widehat{T})} d t+\int_{0}^{1} t^{l-1}(\omega(t \boldsymbol{x})+\pi(t \boldsymbol{x}))(\boldsymbol{x}, \bullet) d t \\
& =k_{0}(\omega)(\boldsymbol{x})(\bullet)+\eta(\boldsymbol{x})(\bullet),
\end{aligned}
$$

with an $\eta \in \mathcal{D P}_{k}^{l-1}(\widehat{T})$ according to Lemma 5 . 
We now scrutinize a few special cases of local ansatz spaces generated by formula (16):

- For $l=n$ we get $\mathcal{X}_{k}^{l}(\widehat{T})=\mathcal{D P}_{k}^{l}(\widehat{T})$, since $\widetilde{\mathcal{H N}}_{k}^{l+1}(\widehat{T})=\{0\}$.

- For any $\omega \in \mathcal{D} \mathcal{F}^{0,1}(\widehat{T})$ we have $\omega=k_{0}(d \omega)$ by (8). Obviously this involves

$$
k_{0}\left(\widetilde{\mathcal{H N}}_{k}^{1}(\widehat{T})\right)=\widetilde{\mathcal{D P}}_{k+1}^{0}(\widehat{T})
$$

as $d \omega \in \widetilde{\mathcal{H N}}_{k}^{1}(\widehat{T})$ for all $\omega \in \widetilde{\mathcal{D P}}_{k+1}^{0}(\widehat{T})$. An easy consequence is that in the case $l=0$

$$
\mathcal{X}_{k}^{0}(\widehat{T})=\mathcal{D P}_{k}^{0}(\widehat{T})+\widetilde{\mathcal{D P}}_{k+1}^{0}(\widehat{T})=\mathcal{D} \mathcal{P}_{k+1}^{0}(\widehat{T}),
$$

i.e. the full space of polynomials of degree $\leq k+1$.

- For $l=n-1$, since $\widetilde{\mathcal{H N}}_{k}^{n}(\widehat{T})=\widetilde{\mathcal{D P}}_{k}^{n}(\widehat{T})$, we end up with

$$
\mathcal{X}_{k}^{n-1}(\widehat{T})=\mathcal{D P}_{k}^{n-1}(\widehat{T})+k_{0}\left(\widetilde{\mathcal{D P}}_{k}^{n}(\widehat{T})\right)
$$

Now consider an $n$-form $\eta \in \widetilde{\mathcal{D P}}_{k}^{n}(\widehat{T})$ of the form $\eta(\boldsymbol{x})=p(\boldsymbol{x}) d x_{1} \wedge \ldots \wedge d x_{n}$ with $p \in \tilde{\mathcal{P}}_{k}(\widehat{T})$. Then we have

$$
\begin{aligned}
k_{0}(\eta)(\boldsymbol{x})\left(\boldsymbol{e}_{1}, \ldots, \check{\boldsymbol{e}}_{j}, \ldots, \boldsymbol{e}_{n}\right) & =\int_{0}^{1} t^{n-1} \eta(t \boldsymbol{x})\left(\boldsymbol{x}, \boldsymbol{e}_{1}, \ldots, \check{\boldsymbol{e}}_{j}, \ldots, \boldsymbol{e}_{n}\right) d t \\
& =\int_{0}^{1} t^{n-1+k} p(\boldsymbol{x})(-1)^{j+1} x_{j} d t \\
& =\frac{1}{n+k} p(\boldsymbol{x})(-1)^{j+1} x_{j} .
\end{aligned}
$$

The natural basis of $\triangle^{n-1}\left(\mathbb{R}^{n}\right)$ is given by

$$
\left\{(-1)^{j+1} d x_{1} \wedge \ldots \wedge \check{d x} x_{j} \wedge \ldots \wedge d x_{n}\right\}_{j=1}^{n} .
$$

From the previous computations we conclude that

$$
k_{0}(\eta)(\boldsymbol{x})=\frac{1}{n+k} p(\boldsymbol{x}) \sum_{j=1}^{n} x_{j}(-1)^{j+1} d x_{1} \wedge \ldots \wedge \check{d} x_{j} \wedge \ldots d x_{n} .
$$

We can use the basis (17) to switch to vector fields. In this context (18) means

$$
\Upsilon_{n-1}^{n}\left(k_{0}(\eta)\right) \in \boldsymbol{x} \cdot \tilde{\mathcal{P}}_{k}(\widehat{T})
$$

Another lesson from (18) is that any such vector field can be created as the image of an $\eta \in \widetilde{\mathcal{D P}}_{k}^{n}(\widehat{T})$ under the potential mapping. All together, we have shown that

$$
\Upsilon_{n-1}^{n}\left(\mathcal{X}_{k}^{n-1}(\widehat{T})\right)=\left(\mathcal{P}_{k}(\widehat{T})\right)^{n}+\boldsymbol{x} \cdot \tilde{\mathcal{P}}_{k}(\widehat{T})
$$

This is the familiar expression for the local Raviart-Thomas spaces (see [28, $26,11])$.

Another special case is that of lowest order finite element approximations, i.e. $k=$ 0. The equality $\widetilde{\mathcal{H N}}_{0}^{l+1}(\widehat{T})=\widetilde{\mathcal{D P}}_{0}^{l+1}(\widehat{T})$ promises simple formulas. In [4] so-called Whitney-forms (a notion developed in [31]) were introduced as a basis for the local ansatz spaces $\mathcal{X}_{0}^{l}(T), l<n$. In $n$-dimensionsal space they are given by

$$
\sum_{j=0}^{l}(-1)^{i_{j}} \lambda_{i_{j}} d \lambda_{i_{0}} \wedge \ldots \wedge d \check{\lambda}_{i_{j}} \wedge \ldots \wedge d \lambda_{i_{l}}, \quad\left\{i_{0}, \ldots, i_{l}\right\} \subset\{0, \ldots, n\},
$$


where $\lambda_{j}$ designates the barycentric coordinate function associated with vertex $j$ of the $n$-simplex $T$. We aim to show that the space of Whitney-forms agrees with $\mathcal{X}_{0}^{l}(T)$.

Again we can return to the reference simplex $\widehat{T}$. There we have $d \lambda_{j}=d x_{j}$ for $j=1, \ldots, n$. Set

$$
\eta:=d x_{j_{1}} \wedge \ldots \wedge d x_{j_{l+1}}, \quad\left\{j_{1}, \ldots, j_{l+1}\right\} \subset\{1, \ldots, n\} .
$$

We now evaluate the potential mapping (7) for $\eta \in \widetilde{\mathcal{D P}}_{0}^{l+1}(\widehat{T})$. To that end we select $\left\{i_{1}, \ldots, i_{l}\right\} \subset\{1, \ldots, n\}$ such that

$$
\begin{aligned}
k_{0}(\eta)(\boldsymbol{x})\left(\boldsymbol{e}_{i_{1}}, \ldots, \boldsymbol{e}_{i_{l}}\right) & =\int_{0}^{1} t^{l} \eta(\boldsymbol{x} t)\left(\boldsymbol{x}, \boldsymbol{e}_{i_{1}}, \ldots, \boldsymbol{e}_{i_{l}}\right) d t \\
& =\frac{1}{l+1} \operatorname{det}\left(\begin{array}{cccc}
x_{j_{1}} & \delta_{i_{1}, j_{1}} & \ldots & \delta_{i_{l}, j_{1}} \\
\vdots & \vdots & & \vdots \\
x_{j_{l+1}} & \delta_{i_{1}, j_{l+1}} & \ldots & \delta_{i_{l}, j_{l+1}}
\end{array}\right) \\
& = \begin{cases}0, & \text { if }\left\{i_{1}, \ldots, i_{l}\right\} \not \subset\left\{j_{1}, \ldots, j_{l+1}\right\}, \\
\pm \frac{1}{l+1} x_{j_{m}}, & \text { if }\left\{i_{1}, \ldots, i_{l}\right\} \cup\left\{j_{m}\right\}=\left\{j_{1}, \ldots, j_{l+1}\right\} .\end{cases}
\end{aligned}
$$

By the definition of the linear forms $d x_{j}$, this means

$$
k_{0}(\eta)(\boldsymbol{x})=\frac{1}{l+1} \sum_{m=1}^{l+1} \pm x_{j_{m}} d x_{j_{1}} \wedge \ldots \wedge \check{d x_{j_{m}}} \wedge \ldots \wedge d x_{j_{l+1}} .
$$

This describes all Whitney-forms whose index set $\left\{i_{0}, \ldots, i_{l}\right\}$ according to (19) does not contain 0 . Choosing another vertex $\boldsymbol{e}_{j}$ of $\widehat{T}$ as the reference point for the potential mapping, we get another collection of Whitney bases with $j$ missing in the index set. Running through all vertices, we thus obtain the entire set of Whitney bases.

So far we know that all Whitney-l-forms on $\widehat{T}$ belong to $\mathcal{X}_{0}^{l}(\widehat{T})$. If there are as many Whitney bases as the dimension of $\mathcal{X}_{0}^{l}(\widehat{T})$, the spaces must agree. Applying elementary combinatorics to (19), we deduce that there are $\left(\begin{array}{c}n+1 \\ l+1\end{array}\right)$ Whitney bases. That this is equal to $\operatorname{dim} \mathcal{X}_{0}^{l}(\widehat{T})$ will be proved right now.

Theorem 6. For $\widehat{T} \subset \mathbb{R}^{n}$ the dimensions of the local ansatz spaces $\mathcal{X}_{k}^{l}(\widehat{T})$ satisfy

$$
\operatorname{dim} \mathcal{X}_{k}^{l}(\widehat{T})=\sum_{i=l}^{n}\left(\begin{array}{c}
n+1 \\
i+1
\end{array}\right)\left(\begin{array}{c}
i \\
i-l
\end{array}\right)\left(\begin{array}{c}
k+l \\
i
\end{array}\right)
$$

for $k \in \mathbb{N}_{0}$ and $0 \leq l \leq n$.

Proof. (I) First we settle the case of 0 -forms. As explained above, $\mathcal{X}_{k}^{0}(\widehat{T})=$ $\mathcal{D} \mathcal{P}_{k+1}^{0}(\widehat{T})$, so that the dimension of $\mathcal{X}_{k}^{0}(\widehat{T})$ is equal to the dimension of the space of multivariate polynomials of degree $\leq k+1$. Thus, the assertion of the theorem for the case $l=0$ follows from (15).

(II) In the general case $l>0$ we first establish a recurrence relation for $\operatorname{dim} \mathcal{X}_{k}^{l}(\widehat{T})$. Our task boils down to figuring out the dimension of $k_{0}\left(\widetilde{\mathcal{H N}}_{k}^{l+1}(\widehat{T})\right)$, because from 
Lemma 5 we learn that $\mathcal{D} \mathcal{P}_{k}^{l}(\widehat{T}) \cap k_{0}\left(\widetilde{\mathcal{H N}}_{k}^{l+1}(\widehat{T})\right)=\emptyset$, and, consequently,

$$
\operatorname{dim} \mathcal{X}_{k}^{l}(\widehat{T})=\operatorname{dim} \mathcal{D} \mathcal{P}_{k}^{l}(\widehat{T})+\operatorname{dim} k_{0}\left(\widetilde{\mathcal{H N}}_{k}^{l+1}(\widehat{T})\right)
$$

We claim that

$$
\widetilde{\mathcal{D P}}_{k+1}^{l}(\widehat{T})=k_{0}\left(\widetilde{\mathcal{H N}}_{k}^{l+1}(\widehat{T})\right) \oplus \widetilde{\mathcal{H N}}_{k+1}^{l}(\widehat{T})
$$

is a direct sum. Too see this, note that due to (8) and Lemma 5 any $\omega \in \widetilde{\mathcal{D P}}_{k+1}^{l}(\widehat{T})$ has such a decomposition. Now, pick $\eta \in k_{0}\left(\widetilde{\mathcal{H N}}_{k}^{l+1}(\widehat{T})\right) \cap \widetilde{\mathcal{H N}}_{k+1}^{l}(\widehat{T})$, that is, $\eta=k_{0}(\omega)$ for a suitable $\omega \in \widetilde{\mathcal{H N}}_{k}^{l+1}(\widehat{T})$. Because $d \eta=\omega=0$ we conclude that $\eta=0$; hence all criteria for a direct sum are satisfied.

In terms of dimensions, (21) means

$$
\operatorname{dim} \widetilde{\mathcal{D P}}_{k+1}^{l}(\widehat{T})=\operatorname{dim} k_{0}\left(\widetilde{\mathcal{H N}}_{k}^{l+1}(\widehat{T})\right)+\operatorname{dim} \widetilde{\mathcal{H N}}_{k+1}^{l}(\widehat{T})
$$

We point out that by Lemma 5 and (8), and since applying the exterior derivative twice results in zero,

$$
\widetilde{\mathcal{H N}}_{k+1}^{l}(\widehat{T})=d\left(\widetilde{\mathcal{D P}}_{k+2}^{l-1}(\widehat{T})\right)
$$

Thanks to the well-known relationship between the rank and the dimension of the null space of the linear mapping $d: \widetilde{\mathcal{D P}}_{k+2}^{l-1}(\widehat{T}) \mapsto \widetilde{\mathcal{H N}}_{k+1}^{l}(\widehat{T})$, we can establish that

$$
\operatorname{dim} \widetilde{\mathcal{H N}}_{k+1}^{l}(\widehat{T})=\operatorname{dim} \widetilde{\mathcal{D P}}_{k+2}^{l-1}(\widehat{T})-\operatorname{dim} \widetilde{\mathcal{H N}}_{k+2}^{l-1}(\widehat{T})
$$

The dimensions of the spaces $\widetilde{\mathcal{D P}}_{k+1}^{l-1}(\widehat{T})$ and $\mathcal{D} \mathcal{P}_{k+1}^{l}(\widehat{T})$ are available as products of the dimension of the spaces of alternating multilinear forms and the dimension of the spaces of the polynomials that occur as coefficient functions. Furthermore, it is clear that $\mathcal{D P}_{k}^{l}(\widehat{T}) \otimes \widetilde{\mathcal{D P}}_{k+1}^{l}(\widehat{T})$ is a direct sum. Then, plugging (22) into (20), we get

$$
\operatorname{dim} \mathcal{X}_{k}^{l}(\widehat{T})=\operatorname{dim} \mathcal{D} \mathcal{P}_{k+1}^{l}(\widehat{T})-\operatorname{dim} \widetilde{\mathcal{H N}}_{k+1}^{l}(\widehat{T})
$$

We rewrite (24) by means of (22) and then rely on (24) itself with $l-1$ instead of $l$. By (15) and addition theorems for binomial coefficients we can derive

$$
\begin{aligned}
\operatorname{dim} \mathcal{X}_{k}^{l}(\widehat{T})= & \operatorname{dim} \mathcal{D P}_{k+1}^{l}(\widehat{T})-\operatorname{dim} \widetilde{\mathcal{D P}}_{k+2}^{l-1}(\widehat{T})+\operatorname{dim} \mathcal{D} \mathcal{P}_{k+2}^{l-1}(\widehat{T})-\operatorname{dim} \mathcal{X}_{k+1}^{l-1}(\widehat{T}) \\
= & \left(\begin{array}{c}
n \\
l
\end{array}\right)\left(\begin{array}{c}
n+k+1 \\
n
\end{array}\right)-\left(\begin{array}{c}
n \\
l-1
\end{array}\right)\left(\begin{array}{c}
n+k+1 \\
n-1
\end{array}\right) \\
& +\left(\begin{array}{c}
n \\
l-1
\end{array}\right)\left(\begin{array}{c}
n+k+2 \\
n
\end{array}\right)-\operatorname{dim} \mathcal{X}_{k+1}^{l-1}(\widehat{T}) \\
= & \left(\begin{array}{c}
n+1 \\
l
\end{array}\right)\left(\begin{array}{c}
n+k+1 \\
n
\end{array}\right)-\operatorname{dim} \mathcal{X}_{k+1}^{l-1}(\widehat{T}) .
\end{aligned}
$$


It remains to confirm that the expression for $\operatorname{dim} \mathcal{X}_{k}^{l}(\widehat{T})$ satisfies the recurrence relation. This amounts to the identity

$$
\begin{aligned}
\sum_{i=l}^{n} & \left(\begin{array}{c}
n+1 \\
n-i
\end{array}\right)\left(\begin{array}{c}
i \\
l
\end{array}\right)\left(\begin{array}{c}
k+l \\
i
\end{array}\right)+\sum_{i=l-1}^{n}\left(\begin{array}{c}
n+1 \\
n-i
\end{array}\right)\left(\begin{array}{c}
i \\
l-1
\end{array}\right)\left(\begin{array}{c}
k+l \\
i
\end{array}\right) \\
& =\left(\begin{array}{c}
n+1 \\
l
\end{array}\right)\left(\begin{array}{c}
n+k+1 \\
n
\end{array}\right),
\end{aligned}
$$

which leads to

$$
\sum_{i=0}^{n}\left(\begin{array}{c}
n+1 \\
i+1
\end{array}\right)\left(\begin{array}{c}
i+1 \\
l
\end{array}\right)\left(\begin{array}{c}
k+l \\
i
\end{array}\right)=\left(\begin{array}{c}
n+1 \\
l
\end{array}\right)\left(\begin{array}{c}
n+k+1 \\
n
\end{array}\right) .
$$

Using summation formulas for binomial coefficients, this equality can be established through lengthy computations, which we are going to skip here.

Lemma 7. Let $\widehat{f}$ denote an $(n-1)$-dimensional "face" of the reference simplex $\widehat{T}$. Then the trace of $\omega \in \mathcal{X}_{k}^{l}(\widehat{T})$ on $f$ belongs to the space $\omega \in \mathcal{X}_{k}^{l}(\widehat{f})$.

Proof. Obviously, the trace of a form in $\mathcal{D} \mathcal{P}_{k}^{l}(\widehat{T})$ on $\widehat{f}$ belongs to $\mathcal{X}_{k}^{l}(\widehat{f})$. So we need only take care of the forms in the image of the potential mapping.

We pick an arbitrary $\boldsymbol{a} \in \widehat{f}$ and make it the reference point in the definition (16). As we have seen, this does not affect the space itself. From the definition of the trace of a differential form it is clear that

$$
\left(k_{\boldsymbol{a}}(\omega)\right)_{\mid \widehat{f}}=k_{\boldsymbol{a}}\left(\omega_{\mid \widehat{f}}\right) .
$$

As $(d \omega)_{\mid \widehat{f}}=d\left(\omega_{\mid \hat{f}}\right)$, the proof of the lemma is finished.

\section{Degrees of FREedom}

Our focus on affine families of finite elements permits us to supply the definition of the local degrees of freedom on the reference simplex $\widehat{T}$ only. We are looking for a set $\left\{\hat{\kappa}_{1}, \ldots, \hat{\kappa}_{N_{k, l}}\right\}, N_{k, l}=\operatorname{dim} \mathcal{X}_{k}^{l}(\widehat{T}), l \in\{0, \ldots, 3\}, k \in \mathbb{N}_{0}$, of linear forms

$$
\hat{\kappa}_{i}: \mathcal{X}_{k}^{l}(\widehat{T}) \mapsto \mathbb{R}, \quad i \in\left\{1, \ldots, N_{k, l}\right\}
$$

that satisfies the requirements:

(D1) Unisolvence: $\left\{\hat{\kappa}_{1}, \ldots, \hat{\kappa}_{N_{k, l}}\right\}$ is a basis of the dual space $\mathcal{X}_{k}^{l}(\widehat{T})^{\prime}$.

(D2) Invariance: Degrees of freedom remain invariant under canonical transformations of differential forms accompanying a transformation of the reference simplex.

(D3) Locality: For any face $\widehat{f}$ of $\widehat{T}$ and $\omega \in \mathcal{X}_{k}^{l}(\widehat{T})$ the trace $\omega_{\mid \widehat{f}}$ is uniquely determined by certain degrees of freedom that depend only on $\omega_{\mid \hat{f}}$.

The first requirement (D1) means that there must be a one-to-one correspondence between the values of the degrees of freedom and the finite element approximation on an element.

The second condition (D2) reflects our interest in affine families of finite elements. It ensures that the arbitrary choice of a reference element has no impact on the final finite element space. It is also vital for the procedure of turning local degrees of freedom into global ones (see [14] for details).

The third condition (D3) must hold in light of the following theorem. It guarantees that the global degrees of freedom obtained from the local ones provide 
sufficiently tight "glue" between the elements to enforce conformity of the finite element approximation.

Theorem 8 ("Patch condition" for differential forms). Let $\Omega$ be partitioned into $\Omega_{1}$ and $\Omega_{2}$ such that $\bar{\Omega}_{1} \cup \bar{\Omega}_{2}=\bar{\Omega}$ and both subregions also possess piecewise smooth boundaries. Then a differential form $\omega \in L_{\mathcal{D} F, l}^{2}(\Omega), l<n$, with $\omega_{\mid \Omega_{i}} \in H\left(B_{l}^{n} ; \Omega_{i}\right)$, $i=1,2$, belongs to $H\left(B_{l}^{n} ; \Omega\right)$ if

$$
\omega_{\mid \Gamma}^{1}=\omega_{\mid \Gamma}^{2} \text { in } L_{\mathcal{D} \mathcal{F}, l}^{2}(\Gamma)
$$

where $\omega^{i}$ stands for the restriction of $\omega$ to $\Omega_{i}, i=1,2$, and $\Gamma:=\bar{\Omega}_{1} \cap \bar{\Omega}_{2}$.

The proof of this theorem follows standard lines using integration by parts. For this reason it can be skipped.

The relationship (6) suggests that the definition of degrees of freedom should rely on integrals of differential forms, in order to guarantee the invariance property (D2). For the sake of locality (D3), some of these integrals should be evaluated over suitable subsimplices (faces, edges and vertices in three dimensions) on the surface of an element. Via these considerations we arrive at the following degrees of freedom:

Definition 9 (Degrees of freedom). For $o \in \mathcal{M}_{i}(\widehat{T}), 0 \leq i \leq n$, let $\left\{\eta_{1, o}, \ldots, \eta_{d, o}\right\}$, $d=\operatorname{dim} \mathcal{D} \mathcal{P}_{k-i+l}^{i-l}(o)$, denote a basis of $\mathcal{D} \mathcal{P}_{k-i+l}^{i-l}(o)$. Then we choose the linear forms $\widehat{\kappa}_{m, o}: \mathcal{X}_{k}^{l}(\widehat{T}) \mapsto \mathbb{R}$ given by

$$
\widehat{\kappa}_{m, o}(\omega):=\int_{o} \omega \wedge \eta_{m, o}, \quad o \in \mathcal{M}_{i}(\widehat{T}), l \leq i \leq n, 1 \leq m \leq d,
$$

as degrees of freedom belonging to $\mathcal{X}_{k}^{l}(\widehat{T})$. The set of all admissible $\widehat{\kappa}_{m, o}$ will be denoted by $\Xi_{k}^{l}(\widehat{T})$.

The invariance of the $\widehat{\kappa}_{m, o}$ with respect to canonical transforms of differential forms is obvious from Theorem 6 . The locality property (D3) can be deduced from Lemma 7 by a simple induction argument: Pick a face $f \in \mathcal{M}_{n-1}(\widehat{T})$. For $o \in \bar{f}$ the values of the degrees of freedom $\widehat{\kappa}_{i, o}(\omega)$ are determined by $\omega_{\mid f}$. Yet, these linear forms $\widehat{\kappa}_{m, o}$ exactly match the degrees of freedom stipulated by Definition 9 for $\mathcal{X}_{k}^{l}(f)$. Taking unisolvence for granted, we see that $\omega_{\mid f}$ is fixed. It remains to settle this very issue of unisolvence:

Lemma 10. Any $\omega \in \mathcal{D P}_{k}^{l}(\widehat{T})$ with vanishing differential $d \omega$, vanishing trace on all of $\partial \widehat{T}$, and which satisfies

$$
\int_{\widehat{T}} \omega \wedge \eta=0, \quad \forall \eta \in \mathcal{D P}_{k-(n-l)}^{n-l}(\widehat{T})
$$

has to be identically 0.

Proof. We first put forth some compact notations: Given an index set $I:=$ $\left\{i_{1}, \ldots, i_{m}\right\} \subset\{1, \ldots, n\}$, we abbreviate $d x_{I}:=d x_{i_{1}} \wedge \ldots \wedge d x_{i_{m}}$. The set $I^{\prime}$ refers to the complement of $I$ in $\{1, \ldots, n\}$. We write $I^{m}$ for the collection of all $m$-subsets of $\{1, \ldots, n\}$.

Pick an $\omega \in \mathcal{D P}_{k}^{l}(\widehat{T})$, which is represented as

$$
\omega=\sum_{I \in I^{l}} \varphi_{I} d x_{I}
$$


and is to satisfy the assumptions of the lemma. Writing $\widehat{f}_{i}$ for the face $\partial \widehat{T} \cap\left\{x_{i}=0\right\}$ of the reference simplex, we know that $\left\{d x_{J}\right\}, J \in I^{l}, i \notin J$, forms a basis of $\triangle^{l}\left(\widehat{f}_{i}\right)$.

Note that

$$
\omega_{\mid \widehat{f}_{i}}=\sum_{I \in I^{l}, i \notin I} \varphi_{I} d x_{I}
$$

Therefore, $\omega_{\mid \widehat{f}_{i}}=0$ implies $\varphi_{I_{\mid \widehat{f}_{i}}}=0$ for all $I \in I^{l}, i \notin I$.

Analogous reasoning for other faces parallel to coordinate hyperplanes yields the following representation for coefficient functions:

$$
\varphi_{I}=\left(\prod_{j \in I^{\prime}} \lambda_{j}\right) \widetilde{\varphi}_{I}
$$

where $\lambda_{j}$ stands for the barycentric coordinate function that is equal to zero on $\widehat{f}_{j}$. Obviously $\widetilde{\varphi}_{I} \in \mathcal{P}_{k-(n-l)}(\widehat{T})$, as the degree of the polynomial shrinks by one for every barycentric coordinate function it is multiplied with.

Now, we select the special test functions $\eta_{I}:=\widetilde{\varphi}_{I} d x_{I^{\prime}}, I \in I^{l}$, Then the expression

$$
\int_{\widehat{T}} \omega \wedge \eta_{I}= \pm \int_{\widehat{T}}\left(\prod_{j \in I^{\prime}} \lambda_{j}\right) \widetilde{\varphi}_{I}^{2} d x_{1} \wedge \ldots \wedge d x_{n}= \pm \int_{\widehat{T}}\left(\prod_{j \in I^{\prime}} \lambda_{j}\right) \widetilde{\varphi}_{I}^{2} d \boldsymbol{x}
$$

has to vanish according to the assumptions of the lemma, as $\eta_{I} \in \mathcal{D} \mathcal{P}_{k-(n-l)}^{n-l}(\widehat{T})$. Thus, necessarily, $\widetilde{\varphi}_{I}=0$. As $I$ is arbitrary, we conclude that $\omega=0$.

Lemma 11. If an $\omega \in \mathcal{X}_{k}^{l}(\widehat{T})$ makes all degrees of freedom given in Definition 9 vanish, then this differential form must be identically zero.

Proof. We employ a "double induction" argument with respect to the dimension $n$ (increasing) and the order $l, 0 \leq l \leq n$, of the differential form (decreasing).

(I) For $n=1$ the assertion of the lemma is trivial. For arbitrary $n$ and $l=n$ we have $\mathcal{X}_{k}^{n}=\mathcal{D P}_{k}^{n}(\widehat{T})$ and only one kind of degree of freedom remaining, namely those of the form

$$
\int_{\widehat{T}} \omega \wedge \eta \text { for } \eta \in \mathcal{D P}_{k}^{0}(\widehat{T}) .
$$

For $\omega=\varphi d x_{1} \wedge \ldots \wedge d x_{n}$ pick $\eta=\varphi$. Then (25) is equal to $\int_{\widehat{T}} \varphi^{2} d \boldsymbol{x}$. Thus the assumption of the lemma immediately implies $\varphi=0$ and $\omega=0$.

(II) Now we admit general $n \in \mathbb{N}$ and $l \in\{0, \ldots, n-1\}$. Assume that the lemma holds true for differential forms of order $l+1$ and in any dimension smaller than $n$.

For any lower dimensional simplex $o \in \mathcal{M}_{i}(\widehat{T}), l+1 \leq i \leq n$, an integration by parts establishes the equality

$$
\int_{o} d \omega \wedge \eta=\int_{o} d(\omega \wedge \eta)-(-1)^{l} \int_{o} \omega \wedge d \eta
$$

with $\eta \in \mathcal{D P}_{k-i+l+1}^{i-l-1}(o)$. The second term must vanish, since, by Lemma $5, d \eta \in$ $\mathcal{D} \mathcal{P}_{k-i+l}^{i-l}(o)$, which makes it belong to the space spanned by the "test polynomials" 
in Definition 9. To the first term we apply Stokes' theorem, and we get

$$
\int_{o} d(\omega \wedge \eta)=\int_{\partial o} \omega \wedge \eta .
$$

Again, we have recovered a right hand side that can be written as a weighted sum of values of degrees of freedom. Hence, the first term must be zero, too.

Recall that, according to Lemma $5, d \omega \in \mathcal{X}_{k}^{l+1}(\widehat{T})$. Above, we have shown that

$$
\int_{o} d \omega \wedge \eta=0 \quad \forall \eta \in \mathcal{D} \mathcal{P}_{k-i+l+1}^{i-l-1}(o), \quad \forall o \in \mathcal{M}_{i}(\widehat{T}), l+1 \leq i \leq n
$$

By the induction assumption with respect to $l$, this enforces $d \omega=0$. This means, for our particular choice of local ansatz spaces, that $\omega \in \mathcal{D} \mathcal{P}_{k}^{l}(\widehat{T})$.

Lemma 7 tells us that $\omega_{\mid \widehat{f}} \in \mathcal{X}_{k}^{l}(\widehat{f})$. As we pointed out before, the degrees of freedom for $\mathcal{X}_{k}^{l}(\widehat{T})$ that belong to a face $\widehat{f}$ are suitable degrees of freedom for $\mathcal{X}_{k}^{l}(\widehat{f})$. Relying on the induction assumption for $n-1$, we see that $\omega_{\mid \partial \widehat{T}}=0$.

In sum, $\omega$ complies with all assumptions of Lemma 10. It teaches us that $\omega=0$. This completes one step of the induction.

Theorem 12 (Unisolvence of degrees of freedom). The degrees of freedom supplied by Definition 9 form a dual basis of $\mathcal{X}_{k}^{l}(\widehat{T})$.

Proof. The previous lemma confirmed that a set of degrees of freedom according to Definition 9 actually spans the dual spaces of $\mathcal{X}_{k}^{l}(\widehat{T})$. To verify that the degrees of freedom are linearly independent, we need only compare their number to the dimension of $\mathcal{X}_{k}^{l}(\widehat{T})$ that is given by Theorem 6 . If we can confirm equality, the proof is finished.

A simple counting argument lets us determine the number of degrees of freedom:

$$
\operatorname{card}\left(\Xi_{k}^{l}(\widehat{T})\right)=\sum_{i=l}^{n}\left(\begin{array}{c}
n+1 \\
i+1
\end{array}\right)\left(\begin{array}{c}
i \\
i-l
\end{array}\right)\left(\begin{array}{c}
k+l \\
i
\end{array}\right)
$$

The first binomial coefficient is the number of simplices of dimension $i$ that are a part of $\partial \widehat{T}$. The second factor gives us the dimension of the spaces of alternating $(i-l)$-multilinear forms in $\mathbb{R}^{i}$. The last factor is the dimension of the polynomial spaces $\mathcal{P}_{k-i+l}(o)$, where $o$ is an $i$-dimensional simplex in $\mathcal{M}_{i}(\widehat{T})$.

As $(26)$ agrees with the expression for $\operatorname{dim} \mathcal{X}_{k}^{l}(\widehat{T})$ from Theorem 6 , we are finished.

Now, we investigate how the newly defined degrees of freedom can be recast as functionals on functions and vector fields in three dimensions:

- For $\mathcal{S}_{k+1}(\widehat{T}):=\Upsilon_{0}^{3}\left(\mathcal{X}_{k}^{0}(\widehat{T})\right), k \in \mathbb{N}_{0}$, the classical Lagrangian finite element, Definition 9 motivates a somewhat nonstandard choice of degrees of freedom 
(see also [18], Definition 3.3): For $\varphi \in \mathcal{S}_{k+1}(\widehat{T})$

$$
\begin{array}{rll}
\hat{\kappa}(\varphi):=\varphi(x) & \text { for } \boldsymbol{x} \in \mathcal{M}_{0}(\widehat{T}), \\
\hat{\kappa}(\varphi):=\int_{\hat{e}} p \cdot \varphi d \Gamma & \text { for } \hat{e} \in \mathcal{M}_{1}(\widehat{T}) \text { and suitable } p \in \mathcal{P}_{k-1}(\hat{e}), \\
\hat{\kappa}(\varphi):=\int_{\hat{f}} p \cdot \varphi d \sigma & \text { for } \hat{f} \in \mathcal{M}_{2}(\widehat{T}) \text { and suitable } p \in \mathcal{P}_{k-2}(\hat{f}), \\
\hat{\kappa}(\varphi):=\int_{\widehat{T}} p \cdot \varphi d \boldsymbol{x} & \text { for suitable } p \in \mathcal{P}_{k-3}(\widehat{T}) .
\end{array}
$$

That these degrees of freedom are $\mathcal{S}_{k+1}(\widehat{T})$-unisolvent is a standard result.

- In the case of Nédélec's spaces $\mathcal{N D}_{k+1}(\widehat{T}):=\Upsilon_{1}^{3}\left(\mathcal{X}_{k}^{1}(\widehat{T})\right)$ (see [26]), we can convert the formulas of Definition 9 into degrees of freedom of the following form (see [19], Definition 5.1): For $\boldsymbol{\xi} \in \mathcal{N}_{\mathcal{D}_{k+1}}(\widehat{T})$

$$
\begin{aligned}
\hat{\kappa}(\boldsymbol{\xi}) & :=\int_{\hat{e}} p \cdot\langle\boldsymbol{\xi}, \boldsymbol{t}\rangle d \Gamma \quad & \text { for } \hat{e} \in \mathcal{M}_{1}(\widehat{T}) \text { and suitable } p \in \mathcal{P}_{k}(\hat{e}), \\
\hat{\kappa}(\boldsymbol{\xi}) & :=\int_{\hat{f}}\langle\boldsymbol{p}, \boldsymbol{\xi} \times \boldsymbol{n}\rangle d \sigma & \text { for } \hat{f} \in \mathcal{M}_{2}(\widehat{T}) \text { and suitable } \boldsymbol{p} \in\left(\mathcal{P}_{k-1}(\hat{f})\right)^{2}, \\
\hat{\kappa}(\boldsymbol{\xi}) & :=\int_{\widehat{T}}\langle\boldsymbol{p}, \boldsymbol{\xi}\rangle d \boldsymbol{x} & \text { for suitable } \boldsymbol{p} \in\left(\mathcal{P}_{k-2}(\widehat{T})\right)^{3} .
\end{aligned}
$$

For a vector-analytic proof of $\mathcal{N} \mathcal{D}_{k+1}(\widehat{T})$-unisolvence, see [26].

- According to [11], for Raviart-Thomas spaces $\Upsilon_{2}^{3}\left(\mathcal{X}_{k}^{2}(\widehat{T})\right)$ the appropriate degrees of freedom are

$$
\begin{aligned}
\hat{\kappa}(\boldsymbol{u}):=\int_{\hat{f}} p\langle\boldsymbol{u}, \boldsymbol{n}\rangle d \sigma \quad \text { for } \hat{f} \in \mathcal{M}_{2}(\widehat{T}) \text { and suitable } p \in \mathcal{P}_{k}(\hat{f}), \\
\hat{\kappa}(\boldsymbol{u}):=\int_{\widehat{T}}\langle\boldsymbol{p}, \boldsymbol{u}\rangle d \boldsymbol{x} \quad \text { for suitable } \boldsymbol{p} \in\left(\mathcal{P}_{k-1}(\widehat{T})\right)^{3} .
\end{aligned}
$$

They fit Definition 9 expressed in terms of vector fields.

- The degrees of freedom for $\Upsilon_{3}^{3}\left(\mathcal{X}_{k}^{3}(\widehat{T})\right)$ are trivial:

$$
\hat{\kappa}(w):=\int_{\widehat{T}} p \cdot w d \boldsymbol{x} \quad \text { for suitable } p \in \mathcal{P}_{k}(\widehat{T}) .
$$

By the customary affine equivalence technique (cf. [8], Chapter 3) we now get degrees of freedom for a simplex with arbitrary shape. With degrees of freedom at our disposal we are now able to define conforming finite element subspaces $\mathcal{X}_{k}^{l}\left(\Omega, \mathcal{T}_{h}\right)$ of $H\left(B_{l}^{n} ; \Omega\right)$ based on the simplicial triangulation $\mathcal{T}_{h}$. They are completely characterized by the following two natural requirements:

- The restriction of any differential form in $\mathcal{X}_{k}^{l}\left(\Omega ; \mathcal{T}_{h}\right)$ to $T$ belongs to $\mathcal{X}_{k}^{l}(T)$.

- The values of degrees of freedom shared by several elements, i.e. those located on $o \in \mathcal{M}_{i}(T)$ for $i<n$, are uniquely defined for every $\omega_{h} \in \mathcal{X}_{k}^{l}\left(\Omega ; \mathcal{T}_{h}\right)$.

Owing to Theorem 8 and Lemma 7 , the second condition ensures $H\left(B_{l}^{n} ; \Omega\right)$ conformity. 
Given global degrees of freedom, canonical interpolation operators (nodal projectors)

$$
\Pi_{\Omega, \mathcal{T}_{h}}^{\mathcal{X}_{k}^{l}}: \mathcal{D} \mathcal{F}^{l, 0}(\Omega) \mapsto \mathcal{X}_{k}^{l}\left(\Omega ; \mathcal{T}_{h}\right)
$$

are also declared by assigning to a continuous function that unique finite element function with the same nodal values.

An all-important consequence of the choice of the global degrees of freedom is the "commuting diagram property" (see [11], §II.2, [18], Section 3, and [16]),

Theorem 13 (Commuting diagram property). Given the above definitions of the spaces and the degrees of freedom, the following diagram commutes:

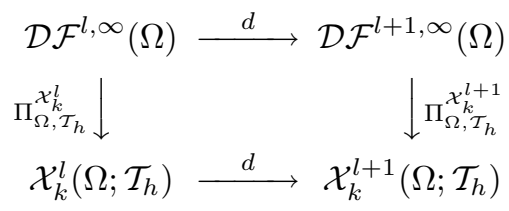

Remark 14. Unfortunately the nodal interpolation operators are not defined for all differential forms in the continuous spaces $L_{\mathcal{D} \mathcal{F}, l}^{2}(\Omega)$. Therefore, we had to confine ourselves to smooth differential forms in the formulation of the theorem.

Proof. To begin with, we remark that the statement of the theorem is purely local, so proving it for the reference element $\widehat{T}$ will do.

For $\omega \in \mathcal{D F}^{l, \infty}(\widehat{T}), 0 \leq l<n$, we set $\pi:=\omega-\Pi_{\widehat{T}_{k}^{l}}^{\widehat{\mathcal{X}^{l}}} \omega$. By the definition of the nodal interpolation operator we see that for $o \in \mathcal{M}_{i}(\widehat{T})$

$$
\int_{o} \pi \wedge \eta=0 \quad \text { for } \eta \in \mathcal{D} \mathcal{P}_{k-i+l}^{i-l}(o), l \leq i \leq n .
$$

The remainder of the proof relies on the same ideas as the proof of Theorem 11 . Integrating by parts, we get, for $\eta \in \mathcal{D} \mathcal{P}_{k-i+l+1}^{i-l-1}(o), l+1 \leq i \leq n, o \in \mathcal{M}_{i}(\widehat{T})$,

$$
\int_{o} d \pi \wedge \eta=\int_{o} d(\pi \wedge \eta)-(-1)^{l} \int_{o} \pi \wedge d \eta .
$$

The second integral evaluates to zero as $d \eta \in \mathcal{D P}_{k-i+l}^{i-l}(o)$. The first term is eligible for an application of Stokes' theorem:

$$
\int_{o} d(\pi \wedge \eta)=\int_{\partial o} \pi \wedge \eta .
$$

Obviously $\partial o \in \mathcal{M}_{i-1}(\hat{T})$ for $o \in \mathcal{M}_{i}(\hat{T})$, so that (27) also forces the first term to vanish. Thus

$$
\Pi_{\widehat{T}}^{\widehat{\mathcal{X}}_{k}^{l+1}} d\left(\omega-\Pi_{\widehat{T}_{k}}^{\hat{\mathcal{X}}^{l}} \omega\right)=0,
$$

and, since $d\left(\Pi_{\widehat{T}}^{\widehat{\mathcal{X}}_{k}^{l}} \omega\right) \in \mathcal{X}_{k}^{l+1}$, we obtain through the unisolvence of the degrees of freedom

$$
\Pi_{\widehat{T}}^{\widehat{\mathcal{X}}_{k}^{l+1}}(d \omega)=d\left(\Pi_{\widehat{T}}^{\widehat{\mathcal{X}}_{k}^{l}} \omega\right)
$$


Taking into account that nodal values are left unchanged by canonical affine transformations, we get (28) for every element and, finally, for the entire finite element spaces.

Two immediate consequences of this theorem are worth mentioning:

Corollary 15. The nodal interpolation operators preserve the kernels of the external derivative, i.e.

$$
d \omega=0 \text { for } \omega \in \mathcal{X}_{k}^{l}\left(\Omega, \mathcal{T}_{h}\right) \quad \Longrightarrow \quad d\left(\Pi_{\Omega, \mathcal{T}_{h}}^{\mathcal{X}_{k}^{l}} \omega\right)=0
$$

The second corollary has to do with the p-hierarchical splitting of higher order finite element spaces. It is naturally induced by the family of nodal interpolation operators parameterized by the polynomial order $k$. Denote the $p$-hierarchical components by

$$
\mathcal{X}_{k}^{l, \mathrm{HB}}\left(\Omega ; \mathcal{T}_{h}\right):=\left(\Pi_{\Omega, \mathcal{T}_{h}}^{\mathcal{X}_{k}^{l}}-\Pi_{\Omega, \mathcal{T}_{h}}^{\mathcal{X}_{k-1}^{l}}\right) \mathcal{X}_{k}^{l}\left(\Omega ; \mathcal{T}_{h}\right) \quad(k \geq 1) .
$$

Corollary 16. With the notations introduced above we have

$$
d \mathcal{X}_{k}^{l, \mathrm{HB}}\left(\Omega ; \mathcal{T}_{h}\right) \subset \mathcal{X}_{k}^{l+1, \mathrm{HB}}\left(\Omega ; \mathcal{T}_{h}\right)
$$

i.e. the external derivative respects the p-hierarchical splitting.

Both corollaries play a central role in the design and the analysis of multilevel schemes for vector valued finite elements (see e.g. [17, 29, 21]), as well as in the derivation of a posteriori error estimators (see [23]).

\section{Discrete Representation theorem}

Poincaré's lemma (Lemma 3) and its analogues for function spaces are tremendously important, since they allow us to represent functions in the kernel of differential operators by means of potentials. A viable discrete model of these function spaces should preserve this property. For any finite element function in the kernel of the relevant differential operator there should exist a suitable potential in another finite element space. Apart from being a beautiful feature, discrete potentials play a crucial role in the design of efficient algorithms for the solution of discretized boundary value problems (see [17, 1, 21, 1, 22, 20, 12]).

It turns out that the family of finite element spaces we obtained from our canonical construction actually provides discrete potentials. To begin with, we show this locally for the reference element. Since canonical transformations and the exterior derivative commute, the result holds for an arbitrary element.

Lemma 17. Let $\widehat{f}_{i}, 0 \leq i \leq n$, denote the faces of the reference simplex $\widehat{T} \in \mathbb{R}^{n}$. Let $\omega \in \mathcal{X}_{k}^{l}(\widehat{T}), 0 \leq l \leq n$, have zero trace on some or all faces of $\widehat{T}$ and vanishing exterior derivative $d \omega$. Then there exists an $\eta \in \mathcal{X}_{k}^{l-1}(\widehat{T})$ whose trace vanishes on the same faces and with the property that $d \eta=\omega$.

Proof. The case that $\omega_{\mid \widehat{f}_{i}}=0$ for all faces $\widehat{f}_{i}$ is the most difficult and will be treated here. This means that $\omega_{\mid \partial \widehat{T}}=0$.

The proof is based on an induction argument with respect to the dimension $n$. For $n=1$ the assertion is trivially correct.

The potential mapping $k_{0}$ from (8) gives a $\pi:=k_{0}(\omega)$ with $d \pi=\omega$. Moreover, by $(16), \pi$ is an element of $\mathcal{X}_{k}^{l-1}(\widehat{T})$. From the very definition of $k_{0}$ and that of 
the trace of a differential form it is clear that $\pi_{\mid \widehat{f}_{i}}=0$ for all faces $\widehat{f}_{i}$ that have the origin 0 as a vertex. There is only a single face $\widetilde{f}$ which does not contain 0 .

In general, $\pi_{\mid \tilde{f}} \neq 0$, but we know that $d \pi_{\mid \tilde{f}}=0$ and $\pi_{\mid \partial \tilde{f}}=0$. Now set $\tau:=\pi_{\mid \tilde{f}}$. Then, according to Lemma $7, \tau$ belongs to $\mathcal{X}_{k}^{l-1}(\tilde{f})$. As $d \tau=0$, by the induction hypotheses, there exists a $\sigma \in \mathcal{X}_{k}^{l-2}(\widetilde{f})$ such that $\sigma_{\mid \partial \tilde{f}}=0$ and $d \sigma=\tau$ on $\widetilde{f}$.

Now, let all degrees of freedom of $\mathcal{X}_{k}^{l-2}(\widehat{T})$ associated with $\tilde{f}$ be determined by $\sigma$. Note that degrees of freedom attached to parts of $\partial \widetilde{f}$ evaluate to zero for $\sigma$. In addition, we set the remaining degrees of freedom in $\Xi_{k}^{l-2}(\widehat{T})$ equal to zero. Thanks to the unisolvence of the degrees of freedom, this defines a unique differential form $\nu$ in $\mathcal{X}_{k}^{l-2}(\widehat{T})$, with $\nu_{\mid \widetilde{f}}=\sigma$ and $\nu_{\mid \partial \widehat{T} / \widetilde{f}}=0$. In particular, the trace of $d \tau$ has to vanish on all faces adjacent to 0 .

Set $\eta:=\pi-d \nu$. Then $d \eta=\omega$ and $\eta_{\mid \widehat{f}_{i}}=0$, provided that the face $\widehat{f}_{i}$ has 0 as vertex. On $\tilde{f}$ we have

$$
\eta_{\mid \tilde{f}}=\pi_{\tilde{f}}-d \nu_{\mid \tilde{f}}=\tau-d \sigma=0 .
$$

In short, we have found a potential $\eta$ of $\omega$ with the desired properties. This carries the induction one dimension further.

The validity of the global version of the discrete representation theorem is confined to simply connected domains. It shares this drawback with its continuous counterparts (compare [19], Chapter 1).

Theorem 18 (Existence of discrete potentials). Let $\Omega \subset \mathbb{R}^{n}$ be a bounded domain with simply connected polyhedral boundary, whose closure is simply connected. $\Omega$ should be endowed with a simplicial triangulation $\mathcal{T}_{h}$. Then for any $\omega \in \mathcal{X}_{k}^{l}\left(\Omega ; \mathcal{T}_{h}\right)$ with $d \omega=0$ we can find an $\eta \in \mathcal{X}_{k}^{l-1}\left(\Omega ; \mathcal{T}_{h}\right)$ such that $\omega=d \eta$.

In addition, if $\omega$ has zero trace on one simply connected part $\Gamma$ of $\partial \Omega$ which can be written as a union of faces of elements, then $\eta$ can have vanishing trace on $\Gamma$, as well.

Proof. It is easy to see that the domain remains simply connected if a simplex $T$ that has at least one face on the boundary $\partial \Omega$ is removed: There is a continuous deformation of $\Omega$ into $\Omega / T$.

The proof employs an induction argument with respect to the number of elements in $\mathcal{T}_{h}$. The case of a single element has already been settled by Lemma 17.

Now, consider a triangulation $\mathcal{T}_{h}$ of $\Omega$ comprising $n$ elements, $n>1$. Remove a simplex $T \in \mathcal{T}_{h}$ that meets the following requirements:

- $T$ is adjacent to the boundary $\partial \Omega$.

- If $\Gamma \neq \emptyset$, then at least one face of $T$ should belong to $\Gamma$. On the other hand, $\bar{T} \cap \Gamma$ should verge on the boundary of $\Gamma$ in $\partial \Omega$, if $\Gamma \neq \partial \Omega$.

There is no doubt that such a $T$ can always be found.

The removal of $T$ creates another simply connected domain $\Omega^{\prime}$, which is triangulated by $n-1$ elements. Set $\Gamma^{\prime}:=\Gamma \cap \partial \Omega^{\prime}$ and note that $\Gamma^{\prime}$ is also simply connected, if nonempty.

By the induction hypotheses, we can find $\pi^{\prime} \in \mathcal{X}_{k}^{l-1}\left(\Omega^{\prime}, \mathcal{T}_{h}\right)$ such that $d \pi^{\prime}=\omega$ in $\Omega^{\prime}$ and $\pi_{\mid \Gamma^{\prime}}=0$. At this stage, we have to exploit the assumption that $\overline{\Omega^{\prime}}$ is simply connected. This assumption makes sure that $\pi^{\prime}$ has a well defined trace onto $\partial T \cap \partial \Omega^{\prime}$. As a consequence, all degrees of freedom located on $\partial T \cap \partial \Omega^{\prime}$ give a well 


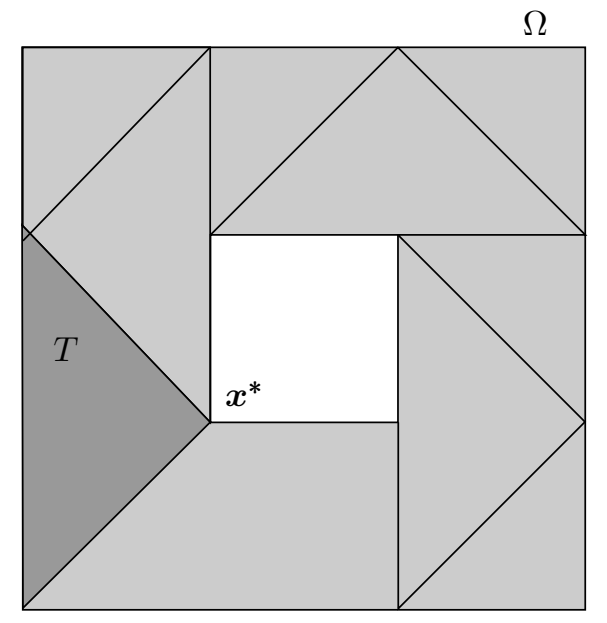

FiguRe 1. Impossible continuation at $\boldsymbol{x}^{*}$

defined value for $\pi^{\prime}$. Setting the remaining degrees of freedom associated with $T$ equal to zero yields a valid extension of $\pi^{\prime}$ to a differential form $\pi \in \mathcal{X}_{k}^{l-1}\left(\Omega, \mathcal{T}_{h}\right)$.

But $\sigma:=\omega-d \pi$ can be nonzero only on $T$ and has vanishing trace on $\partial T \cap \partial \Omega^{\prime}$ and $\partial T \cap \Gamma$. According to the previous lemma there exists a $\tau \in \mathcal{X}_{k}^{l-1}(T)$ with $d \tau=\sigma-d \pi$ and zero trace on the same faces as $\sigma$. This property of $\tau_{\mid \partial T}$ permits us to extend $\tau$ by zero to an element of $\mathcal{X}_{k}^{l-1}\left(\Omega ; \mathcal{T}_{h}\right)$ (also designated by $\tau$ ). Obviously $\eta:=\pi+\tau \in \mathcal{X}_{k}^{l-1}\left(\Omega, \mathcal{T}_{h}\right)$ supplies the desired potential.

Remark 19. A simple example can illustrate why it is significant that $\bar{\Omega}$ is simply connected. Consider the 2D situation sketched in Figure 1 and the case $l=1$. Thus, we seek discrete potentials among plain scalar Lagrangian finite element functions. Evaluating them at vertices provides the most basic degrees of freedom that Definition 9 describes. Now, there might exist a well-defined potential $\pi^{\prime}$ for $\omega$ on $\Omega^{\prime}:=\Omega / T$. Nevertheless, it is by no means guaranteed that $\pi^{\prime}$ can be continuously extended onto $\bar{\Omega}^{\prime}$; inconsistent values in $\boldsymbol{x}^{*}$ might emerge! Obviously, this rules out a continuation of $\pi^{\prime}$ onto the entire domain $\Omega$.

\section{Conclusion}

In this paper we have developed a fully canonical construction of simplicial finite elements, which are conforming with respect to spaces related to differential operators from vector analysis. Previously, only separate, seemingly unrelated, schemes in two and three dimensions were available. Moreover, to prove the validity of these approaches required a significant technical effort in each case. Our theory offers an elegant remedy: a single proof that covers all cases. Moreover, important results like the discrete representation theorem and the commuting diagram property had not been established in full generality before. All this could be achieved by an unprecedented application of the theory of differential forms in the mathematical analysis of algebraic aspects of finite element schemes. 


\section{REFERENCES}

[1] D. Arnold, R. Falk, and R. Winther, Preconditioning in $H$ (div) and applications, Math. Comp., 66 (1997), pp. 957-984. MR 97i:65177

[2] D. BALDOMIR, Differential forms and electromagnetism in 3-dimensional Euclidean space $\mathbb{R}^{3}$., IEE Proc. A, 133 (1986), pp. 139-143. CMP 18:17

[3] A. Bossavit, A rationale for edge elements in 3D field computations, IEEE Trans. Mag., 24 (1988), pp. $74-79$.

[4] - Whitney forms: A class of finite elements for three-dimensional computations in electromagnetism, IEE Proc. A, 135 (1988), pp. 493-500.

[5] —- A new viewpoint on mixed elements, Meccanica, 27 (1992), pp. 3-11.

[6] __ Électromagnétisme, en vue de la modélisation, Springer-Verlag, Paris, 1993. CMP 98:11

[7] — Computational Electromagnetism. Variational Formulation, Complementarity, Edge Elements, no. 2 in Academic Press Electromagnetism Series, Academic Press, San Diego, 1998. CMP 98:06

[8] S. Brenner And R. Scott, Mathematical theory of finite element methods, Texts in Applied Mathematics, Springer-Verlag, New York, 1994. MR 95f:65001

[9] F. Brezzi, J. Douglas, R. Duran, and M. Fortin, Mixed finite elements for 2nd order elliptic problems in three variables, Numer. Math., 51 (1987), pp. 237-250. MR 88f:65190

[10] F. Brezzi, J. Douglas, M. Fortin, and D. Marini, Efficient rectangular mixed finite elements in two and three space variables, RAIRO Modél. Math. Anal. Numér. 21 (1987), pp. 581-604. MR 88j:65249

[11] F. Brezzi And M. Fortin, Mixed and hybrid finite element methods, Springer-Verlag, New York, 1991. MR 92d:65187

[12] Z. Cai, R. Parashkevov, T. Russel, and X. Ye, Domain decomposition for a mixed finite element method in three dimensions, in Proc. 9th Internat. Conf. Domain Decomposition Methods, Bergen, Norway, 1996 (to appear).

[13] H. Cartan, Formes Différentielles, Hermann, Paris, 1967. MR 37:6858

[14] P. Ciarlet, The Finite Element Method for Elliptic Problems, North-Holland, Amsterdam, 1978. MR 58:25001

[15] G. Deschamps, Electromagnetics and differential forms, Proc IEEE, 69 (1981), pp. 676-695.

[16] J. Douglas and J. Roberts, Global estimates for mixed methods for 2nd order elliptic equations, Math. Comp., 44 (1985), pp. 39-52. MR 86b:65122

[17] R. EWIng AND J. WANG, Analysis of the Schwarz algorithm for mixed finite element methods, RAIRO Modél. Math. Anal. Numér., 26 (1992), pp. 739-756. MR 94c:65135

[18] V. GiRault, Curl-conforming finite element methods for Navier-Stokes equations with nonstandard boundary conditions in $\mathbb{R}^{3}$, vol. 1431 of Lecture Notes in Mathematics, SpringerVerlag, Berlin, 1989, pp. 201-218. MR 91k:65143

[19] V. Girault and P. Raviart, Finite element methods for Navier-Stokes equations, SpringerVerlag, Berlin, 1986. MR 88b:65129

[20] R. HiptmaiR, Multigrid method for Maxwell's equations, SIAM J. Numer. Anal. 36 (1999), 204-225. CMP 99:04

[21] R. Hiptmair And R. Hoppe, Multilevel preconditioning for mixed problems in three dimensions, Tech. Rep. 359, Mathematisches Institut, Universität Augsburg, 1996. to appear in Numer. Math.

[22] R. Hiptmair And A. Toselli, Overlapping and multilevel Schwarz methods for vector valued elliptic problems in three dimensions., in Parallel Solution of PDEs, IMA Volumes in Mathematics and its Applications, Springer, Berlin, 1998. to appear.

[23] R. Hoppe And B. Wohlmuth, A comparison of a posteriori error estimators for mixed finite elements, Tech. Rep. 350, Math.-Nat. Fakultät, Universität Augsburg, 1996. to appear in Math. Comp.

[24] E. KaAsschieter And A. HuiJBen, Mixed-hybrid finite element and streamline computation for the potential flow problem, Numer. Methods Partial Differential Equations 8 (1992), pp. 221-266. MR 93b:65169

[25] K. Mahadevan and R. Mitta, Use of Whitney's edge and face elements for efficient finite element time domain solution of Maxwell's equations, J. Electromagn. Waves Appl., 8 (1994), pp. 1173-1191. 
[26] J. NÉDÉLEC, Mixed finite elements in $R^{3}$, Numer. Math., 35 (1980), pp. 315-341. MR 81k:65125

[27] — A new family of mixed finite elements in $R^{3}$, Numer. Math., 50 (1986), pp. 57-81. MR 88e:65145

[28] P. A. Raviart and J. M. Thomas, A Mixed Finite Element Method for Second Order Elliptic Problems, vol. 606 of Springer Lecture Notes in Mathematics, Springer-Verlag, New York, 1977, pp. 292-315. MR 58:3547

[29] P. Vassilevski and J. Wang, Multilevel iterative methods for mixed finite element discretizations of elliptic problems, Numer. Math., 63 (1992), pp. 503-520. MR 93j:65187

[30] A. Walsleben, Whitney Elemente zur Diskretisierung der Maxwell-Gleichungen, Master's thesis, Institut für Mathematik I, Freie Universität Berlin, 1996.

[31] H. Whitney, Geometric Integration Theory, Princeton Univ. Press, Princeton, 1957. MR 19:309c

Sonderforschungsbereich 382, Universität Tübingen, 72076 Tübingen, Germany

E-mail address: hiptmair@na.uni-tuebingen.de 